\title{
Comment on "Vibrational and configurational parts of the specific heat at glass formation"
}

\author{
Daniele Cangialosi, ${ }^{1, *}$ Angel Alegría, ${ }^{1,2}$ and Juan Colmenero ${ }^{1,2,3}$ \\ ${ }^{1}$ Centro de Física de Materiales, CSIC-UPV/EHU, Edificio Korta, 20018 San Sebastián, Spain \\ ${ }^{2}$ Departamento de Física de Materiales, Facultad de Química, Universidad del País Vasco (UPV/EHU), Apartado 1072, \\ 20080 San Sebastián, Spain \\ ${ }^{3}$ Donostia International Physics Center, Paseo Manuel de Lardizabal 4, 20018 San Sebastián, Spain
}

(Received 23 January 2008; published 20 November 2008)

\begin{abstract}
The result found in a recent paper [Tombari et al., Phys. Rev. B 77, 024304 (2008)] regarding the relatively small contribution of secondary relaxation processes to the specific heat is discussed with reference to the claimed non-negligible contribution of the same processes to the total excess entropy found in another paper [Cangialosi et al., Europhys. Lett. 70, 614 (2005)]. We provide indications that the two results are consistent with each other.
\end{abstract}

DOI: 10.1103/PhysRevB.78.176301

PACS number(s): 61.20.Lc, 61.43.Fs, 64.70.P-

In a recent paper, Tombari et al. ${ }^{1}$ reported detailed calorimetric measurements on several glass-forming polymers and analyzed their data with the aim of obtaining the different contributions to the specific heat of those polymers. Among the different contributions to the specific heat those related to secondary relaxation processes were found to be relatively small. Inspired by this finding, there arose serious criticism about the validity of the conclusion found by Cangialosi et al., ${ }^{2}$ who-testing the applicability of the AdamGibbs theory ${ }^{3}$ to glass-forming polymers-gave indication that for some glass-forming polymers a significant fraction of the excess entropy at the glass transition temperature $\left(T_{g}\right)$ should be attributed to secondary relaxation processes. In particular, Tombari et al. ${ }^{1}$ claimed that in Cangialosi et al. ${ }^{2}$ "the Adam-Gibbs equation empirically fits the data for polymers if it is assumed that secondary relaxation makes a $50 \%-90 \%$ contribution to the total excess entropy at $T_{g}$ via a very large contribution to $c_{p}$ from the secondary relaxation in polymers." First of all, we remark that such a large contribution to the total excess entropy at $T_{g}$ has been found only for those polymers possessing internal degrees of freedom manifesting with secondary relaxation processes unrelated to the structural relaxation process. Apart from this, in Cangialosi et $a l .{ }^{2}$ it is never claimed that such a large contribution to the excess entropy originates from a very large contribution to $c_{p}$ from the secondary relaxation in polymers. Hereafter we provide indications that the results found by Tombari et al. ${ }^{1}$ not only are not in contradiction with those of Cangialosi et al., ${ }^{2}$ but they are even in agreement.

The specific heat $\left(c_{p}\right)$ is related to the entropy $(S)$ by the following relation:

$$
\left.\frac{\partial S}{\partial T}\right|_{P}=\frac{c_{p}}{T} .
$$

A negligible specific heat was found by Tombari et al. ${ }^{1}$ for secondary relaxations. Taking into account that this result has been found at relatively high temperature (in the range 300-
$400 \mathrm{~K}$ ), also the ratio of a negligible specific heat to temperature is negligible and, therefore, the entropy associated to such a specific heat is roughly temperature independent at constant pressure. This is exactly what has been found in the analysis of Cangialosi et al. ${ }^{2}$ Thus the finding of Tombari et $a l .{ }^{1}$ provides an additional support to the temperature independence of the excess entropy associated with secondary relaxation processes. However, we remark that the temperature independence of that fraction of the excess entropy associated to secondary relaxation processes can also be deduced from the absence of sub- $T_{g}$ jumps in the specific heat vs temperature plots of glass-forming polymers. ${ }^{4}$ In particular, a non-negligible specific heat associated to secondary relaxation processes would give rise to a specific-heat jump at the temperature for which the time scale of the secondary process under consideration approximately equals that of the calorimetric experiment (related to the cooling or heating rate of the experiment itself and, in the case of temperature modulated calorimetric experiments, the frequency). The temperature independence of the entropy associated to secondary relaxation processes related to some internal degree of freedom can be plausibly explained considering that the geometry of the motion associated to those degrees of freedom-and, therefore, the number of configuration that can be explored through such a motion-and its density of state are temperature independent.

Furthermore, it is worth noticing that the analysis conducted by Cangialosi et $a .^{2}$ to evaluate the actual value of the temperature independent excess entropy is not based on the mere fitting of relaxational data above $T_{g}$-as suggested by Tombari et al. ${ }^{1}$ This value, not incidentally, coincides with the residual excess entropy-evaluated from independent calorimetric measurements ${ }^{4}$ - at the Vogel temperature, namely, where any contribution to the excess entropy from the structural relaxation process is predicted to vanish, provided that both the excess entropy and the structural relaxation time are assumed to retain their temperature dependence even below $T_{g}$. 
*swxcacad@sw.ehu.es

${ }^{1}$ E. Tombari, C. Ferrari, G. Salvetti, and G. P. Johari, Phys. Rev. B 77, 024304 (2008).

${ }^{2}$ D. Cangialosi, A. Alegría, and J. Colmenero, Europhys. Lett. 70,
614 (2005).

${ }^{3}$ G. Adam and J. H. Gibbs, J. Chem. Phys. 43, 139 (1965).

${ }^{4}$ B. Wunderlich, Thermal Analysis of Polymeric Materials (Springer, Heidelberg, 2005). 